\title{
Onset of secondary instabilities on the zigzag instability in stratified fluids
}

\author{
PIERRE AUGIER† AND PAUL BILLANT \\ LadHyX, CNRS, Ecole Polytechnique, 91128 Palaiseau Cedex, France \\ (Received 1 February 2011; revised 4 April 2011; accepted 15 May 2011; \\ first published online 29 June 2011)
}

Recently, Deloncle, Billant \& Chomaz (J. Fluid Mech., vol. 599, 2008, p. 229) and Waite \& Smolarkiewicz (J. Fluid Mech., vol. 606, 2008, p. 239) have performed numerical simulations of the nonlinear evolution of the zigzag instability of a pair of counterrotating vertical vortices in a stratified fluid. Both studies report the development of a small-scale secondary instability when the vortices are strongly bent if the Reynolds number $R e$ is sufficiently high. However, the two papers are at variance about the nature of this secondary instability: it is a shear instability according to Deloncle et al. (J. Fluid Mech., vol. 599, 2008, p. 229) and a gravitational instability according to Waite \& Smolarkiewicz (J. Fluid Mech., vol. 606, 2008, p. 239). They also profoundly disagree about the condition for the onset of the secondary instability: $R e F_{h}{ }^{2}>O(1)$ according to the former or $R e F_{h}>80$ according to the latter, where $F_{h}$ is the horizontal Froude number. In order to understand the origin of these discrepancies, we have carried out direct numerical simulations of the zigzag instability of a Lamb-Chaplygin vortex pair for a wide range of Reynolds and Froude numbers. The threshold for the onset of a secondary instability is found to be $\operatorname{Re} F_{h}{ }^{2} \simeq 4$ for $\operatorname{Re} \gtrsim 3000$ and $\operatorname{Re} F_{h}=80$ for $R e \lesssim 1000$ in agreement with both previous studies. We show that the scaling analysis of Deloncle et al. (J. Fluid Mech., vol. 599, 2008, p. 229) can be refined to obtain a universal threshold: $\left(R e-R e_{0}\right) F_{h}{ }^{2} \simeq 4$, with $R e_{0} \simeq 400$, which works for all $R e$. Two different regimes for the secondary instabilities are observed: when $\left(R e-R e_{0}\right) F_{h}{ }^{2} \simeq 4$, only the shear instability develops while when $\left(R e-R e_{0}\right) F_{h}{ }^{2} \gg 4$, both shear and gravitational instabilities appear almost simultaneously in distinct regions of the vortices. However, the shear instability seems to play a dominant role in the breakdown into small scales in the range of parameters investigated.

Key words: stratified flows, transition to turbulence, vortex instability.

\section{Introduction}

Strongly stratified flows are anisotropic and generally exhibit a layered structure (Riley \& Lelong 2000). Layers can arise spontaneously through an instability, the zigzag instability, when several vertical vortices are interacting. This has been shown in the cases of pairs of counter- or co-rotating vortices (Billant \& Chomaz 2000a; Otheguy, Chomaz \& Billant 2006) and vortex arrays (Deloncle, Billant \& Chomaz 2011). The study of such elementary flows is convenient to identify and understand some of the fundamental mechanisms at work in more complicated flows such as stratified turbulence. 
The zigzag instability exists for low horizontal Froude number $F_{h}=U /(N a)$, where $U$ is the typical horizontal velocity of the vortices, $a$ the vortex radius and $N$ the Brunt-Väisälä frequency. It originates from the coupling between vortex bending waves and the strain exerted by companion vortices (Otheguy, Billant \& Chomaz 2007; Billant 2010; Billant et al. 2010). The instability bends the vortices with a growth rate scaling like the strain and a vertical wavelength scaling like $L_{b} b / a$, where $L_{b}=U / N$ is the buoyancy length and $b$ the vortex separation distance.

In the laboratory, the zigzag instability of a counter-rotating vortex pair has been observed to grow to a very large amplitude producing high vertical shear and leading to the formation of thin but laminar layers (Billant \& Chomaz 2000a). Recently, Deloncle et al. (2008) and Waite \& Smolarkiewicz (2008) have carried out numerical simulations of the nonlinear evolution of the zigzag instability at higher Reynolds number $R e=U a / v$ (where $v$ is the viscosity) than in the laboratory. They have both observed that small scales develop leading to a transition to turbulence when the zigzag instability is fully developed and the Reynolds number sufficiently large.

Deloncle et al. (2008) attributed this breakdown into small scales to the onset of the Kelvin-Helmholtz instability by showing that it develops in regions where the Richardson number becomes lower than $1 / 4$. In contrast, Waite \& Smolarkiewicz (2008) have shown that small scales emerge when density perturbations generated by the zigzag instability become gravitationally unstable.

In addition to this difference of interpretation about the nature of the secondary instabilities, Deloncle et al. (2008) and Waite \& Smolarkiewicz (2008) also disagree about the threshold for their onsets. Deloncle et al. (2008) have shown that the exponential growth of the zigzag instability is not saturated by nonlinear effects but by viscous effects due to the vertical shear when there is no secondary instability. This occurs when the vertical length scale $L_{v}$ has decreased down to the viscous length scale $L_{v} \sim a / \sqrt{R e}$. At that time, the Richardson number is $R i \simeq N^{2} /\left|\partial \boldsymbol{u}_{h} / \partial z\right|^{2} \propto$ $N^{2} L_{v}^{2} / U^{2} \propto 1 /\left(R e F_{h}^{2}\right)$, where $\partial \boldsymbol{u}_{h} / \partial z$ is the vertical gradient of horizontal velocity. Therefore, when $R e F_{h}{ }^{2}$ is large, the Richardson number can be lower than 1/4 before viscous saturation of the zigzag instability. Deloncle et al. (2008) have shown that this criterion predicts well the occurrence of the shear instability in their simulations. In contrast, Waite \& Smolarkiewicz (2008) reported that the gravitational instability occurs when $\operatorname{Re} F_{h}>80$. However, this threshold is empirical and no theoretical justification has been provided by Waite \& Smolarkiewicz (2008).

Deloncle et al. (2008) and Waite \& Smolarkiewicz (2008) have speculated that the discrepancies between their conclusions might come from the different initial conditions: Waite \& Smolarkiewicz (2008) used a Lamb-Chaplygin vortex pair while Deloncle et al. (2008) used a pair of counter-rotating Gaussian vortices with a separation distance $b=2.5 a$. In order to check this hypothesis, we have reconsidered the problem and performed direct numerical simulation (DNS) of a Lamb-Chaplygin vortex pair for a wide range of Reynolds number $R e$ and Froude number $F_{h}$.

\section{Numerical method and initial conditions}

The numerical procedure is the same as in Deloncle et al. (2008) except that the vortex pair consists of a Lamb-Chaplygin dipole instead of a pair of adapted counter-rotating Lamb-Oseen vortices. The incompressible Navier-Stokes equations under the Boussinesq approximation are solved by means of a pseudo-spectral method 


\begin{tabular}{llccccc}
\hline \multicolumn{1}{c}{ Run } & $F_{h}$ & $R e$ & $\mathscr{R}=\operatorname{ReF}_{h}{ }^{2}$ & $\mathscr{L}_{x} \times \mathscr{L}_{y} \times \mathscr{L}_{z}$ & $N_{x} \times N_{y} \times N_{z}$ & $\delta t$ \\
Fh0.15Re500 & 0.15 & 400 & 9 & $10 \times 10 \times 1.5$ & $256 \times 256 \times 64$ & 0.01 \\
Fh0.15Re1000 & 0.15 & 1000 & 22.5 & $10 \times 10 \times 1.5$ & $256 \times 256 \times 64$ & 0.01 \\
Fh0.1Re600 & 0.1 & 600 & 6 & $10 \times 10 \times 1.0$ & $256 \times 256 \times 64$ & 0.01 \\
Fh0.1Re2500 & 0.1 & 2500 & 25 & $10 \times 10 \times 1.0$ & $512 \times 512 \times 96$ & 0.005 \\
Fh0.05Re1500 & 0.05 & 1500 & 3.75 & $10 \times 10 \times 0.5$ & $384 \times 384 \times 64$ & 0.006 \\
Fh0.05Re4000 & 0.05 & 4000 & 10 & $10 \times 10 \times 0.5$ & $768 \times 768 \times 96$ & 0.003 \\
Fh0.03Re4500 & 0.03 & 4500 & 4.05 & $10 \times 10 \times 0.3$ & $768 \times 768 \times 96$ & 0.003 \\
Fh0.03Re6000 & 0.03 & 6000 & 5.4 & $10 \times 10 \times 0.3$ & $768 \times 768 \times 96$ & 0.003
\end{tabular}

TABLE 1. Overview of the physical and numerical parameters of the simulations with highest and lowest $R e$ for each $F_{h}$. The number of nodes in the $x$-, $y$ - and $z$-direction are denoted, respectively, by $N_{x}, N_{y}$ and $N_{z}$. The time step is $\delta t$. We recall that the length and time units are $R$ and $R / U$, respectively.

with periodic boundary conditions (see Deloncle et al. (2008) for details). The velocity field $\boldsymbol{u}$ and the density perturbation $\rho^{\prime}$ are initialized as

$$
\left[\boldsymbol{u}, \rho^{\prime}\right](x, y, z, t=0)=\left[\boldsymbol{u}_{2 D}(x, y), 0\right]+\varepsilon \cos \left(2 \pi z / \lambda_{z}\right)\left[\boldsymbol{u}_{p}, \rho_{p}^{\prime}\right](x, y),
$$

where $(x, y, z)$ are Cartesian coordinates with $z$ along the vertical, $\boldsymbol{u}_{2 D}$ is the velocity field of a Lamb-Chaplygin dipole in the co-moving frame like in Waite \& Smolarkiewicz (2008). This dipole is a steady solution of the Euler equation given by $\boldsymbol{u}_{2 D}=-\nabla \times \psi_{0} \boldsymbol{e}_{z}$, with

$$
\psi_{0}(r, \theta) \equiv \begin{cases}\frac{2 U R}{\mu_{1} J_{0}\left(\mu_{1}\right)} J_{1}\left(\mu_{1} r / R\right) \cos \theta, & \text { if } r<R, \\ U r\left(1-\frac{R^{2}}{r^{2}}\right) \cos \theta, & \text { if } r \geqslant R,\end{cases}
$$

where $(r, \theta)$ are cylindrical coordinates with $(x=r \cos \theta, y=\sin \theta), U$ the velocity of propagation in the laboratory frame, $R$ the dipole radius, $J_{0}$ and $J_{1}$ are the zerothand first-order Bessel functions and $\mu_{1}=3.8317$ is the first root of $J_{1}$.

The second term on the right-hand side of (2.1) is a small perturbation with $\left[\boldsymbol{u}_{p}, \rho_{p}^{\prime}\right]$ the most unstable eigenmode of $\boldsymbol{u}_{2 D}$ determined by a numerical linear stability analysis, $\lambda_{z}$ its vertical wavelength and $\varepsilon$ a small amplitude.

The Reynolds number $R e$ and the Froude number $F_{h}$ are based on the initial conditions: $R e=U R / v, F_{h}=U /(N R)$, where $v$ is the kinematic viscosity and $N=\sqrt{-\left(g / \rho_{0}\right)(\mathrm{d} \bar{\rho} / \mathrm{d} z)}$ the Brunt-Väisälä frequency (assumed constant), where $g$ is the gravity, $\rho_{0}$ a reference density and $\bar{\rho}(z)$ the basic density profile. The total density is given by $\rho_{t o t}=\rho_{0}+\bar{\rho}(z)+\rho^{\prime}$. The Schmidt number $S c=v / D$, where $D$ is the mass diffusivity, is set to unity in all runs. For simplicity and without loss of generality, $R$ and $R / U$ are taken, respectively, as length and time units, i.e. are fixed to unity. The density perturbations are non-dimensionalized by $R|\mathrm{~d} \bar{\rho} / \mathrm{d} z|$. The same symbols are kept for the non-dimensional variables.

The parameters of the main runs are summarized in table 1 . The value of the Froude number is kept below the threshold $F_{h}=0.2$, where the zigzag instability is dominant in the case of the Lamb-Chaplygin dipole (Billant \& Chomaz 2000b). For each Froude number investigated, only the runs with the highest and lowest Reynolds numbers are listed in table 1. For the runs with intermediate values of $R e$, the resolution and time step have been chosen between these two limiting cases. 


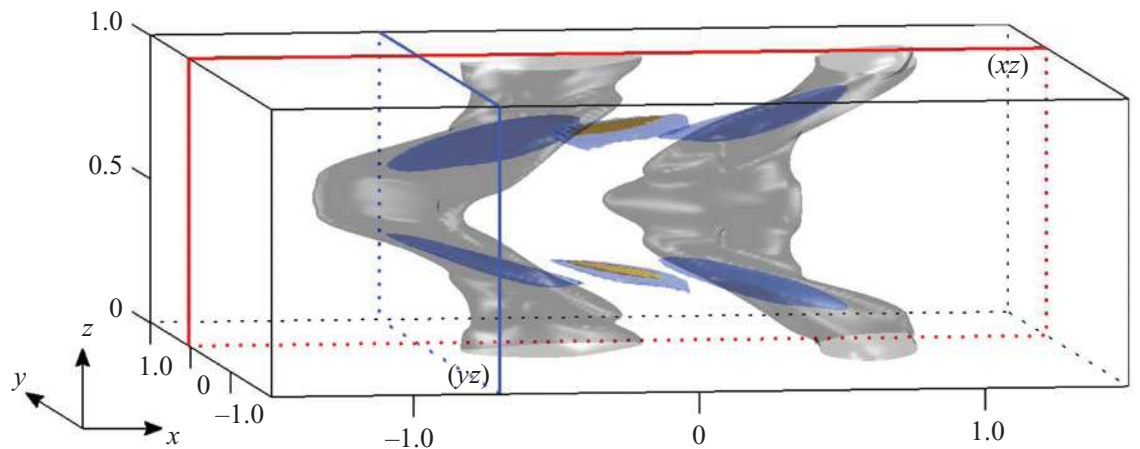

FIGURE 1. Three-dimensional contours of the vertical vorticity of the dipole for $F_{h}=0.1$ and $R e=2500$ at time $t=3.8$. The grey surfaces are iso-surfaces of vertical vorticity $\left|\omega_{z}\right|=0.7 \max \left(\omega_{z}\right)$. The blue surfaces correspond to iso-values of the Richardson number equal to $1 / 4$ (indication of the possibility of shear instability) and the orange surfaces correspond to iso-values $\partial \rho_{t o t} / \partial z=0$ (instability condition for the gravitational instability). The red and blue lines indicate the position of the vertical cross-sections $(x z)$ and $(y z)$ displayed in figure $2(a, b)$.

The height of the computational domain is set to the most amplified wavelength of the zigzag instability $\mathscr{L}_{z}=\lambda_{z}$. The horizontal size of the box $\mathscr{L}_{x}=\mathscr{L}_{y}=10$ is taken sufficiently large compared to the dipole radius $R=1$ in order to have negligible effects of the periodic boundary conditions. Several additional runs have been performed with different domain sizes and numerical resolutions in order to check the accuracy and convergence of the results presented.

\section{Description of the transition to small scales}

We first describe a simulation for $F_{h}=0.1$ and $R e=2500$ for which the nonlinear evolution of the zigzag instability leads to a transition to small scales. Since the development of the zigzag instability has already been described in detail by Waite \& Smolarkiewicz (2008) and Deloncle et al. (2008), we start our description at the time when the zigzag instability is mature and the transition to small scales is incipient. Our main purpose is to determine the nature of the underlying secondary instabilities.

Figure 1 displays in grey the iso-surfaces of vertical vorticity at $t=3.8$. As can be seen, the zigzag instability has reached a finite amplitude and the vortices are fully bent but small scales have not yet developed. Even if the growth of the zigzag instability is still exponential at that time, the bending deformation of the vortices is no longer purely sinusoidal: one can see that the vortices tend to be vertically aligned at the extrema of the bending deformation, i.e. at $z=0$ and $z=0.5$, while in between the vortices tend to be more inclined than for a pure sinusoid. In order to identify regions susceptible to the shear instability, we have plotted in blue the iso-surfaces where the local Richardson number

$$
R i=\frac{-\partial \rho_{t o t} / \partial z}{F_{h}{ }^{2}\left|\partial \boldsymbol{u}_{h} / \partial z\right|^{2}}
$$

is equal to $1 / 4$, where $\partial \rho_{\text {tot }} / \partial z$ is the non-dimensional vertical gradient of total density, i.e. $\partial \rho_{\text {tot }} / \partial z=-1+\partial \rho^{\prime} / \partial z$. The condition $R i<1 / 4$ somewhere in the flow is a necessary but not sufficient condition for the shear instability of a steady parallel inviscid shear flow (Howard 1961; Miles 1961). It is therefore not rigorously applicable to the present 
flow but we expect that the application of this condition will be meaningful if the flow is sufficiently parallel and evolves sufficiently slowly compared, respectively, to the characteristic length scale and time scale of the shear instability. As seen in figure 1 , there are six distinct regions where $R i<1 / 4$. Four of these regions are located close to the iso-vorticity surfaces, i.e. inside the vortex cores at the vertical levels where they are most slanted. The two other regions are located approximately at the same vertical levels but right in the middle between the two vortex cores. Quite strikingly, an orange surface, corresponding to the iso-value $R i=0$, is embedded within each of the two latter regions. This means that the vertical gradient of the total density is positive inside these regions so that they are prone to a convective instability. Interestingly, the conditions for the shear and gravitational instabilities can be also satisfied simultaneously for inertia-gravity waves (see, e.g. Lelong \& Dunkerton 1998; Fritts \& Alexander 2003). However, we emphasize that here these two conditions are fulfilled for distinct physical reasons: the regions where $0<R i<1 / 4$ appear in the vortex cores because the bending of the vortices enhances the vertical shear (Deloncle et al. 2008). In contrast, the regions where $R i<0$ (connected to small regions where $R i<1 / 4)$ appear because the bending of the low-pressure vortex cores produces high vertical pressure gradient and so high-density perturbations by hydrostatic balance (Waite \& Smolarkiewicz 2008).

The precise position of these regions can be seen more clearly in figures $2(a)$ and $2(b)$ which show the colour contours of the Richardson number in the vertical cross-sections $y z$ and $x z$ indicated, respectively, in blue and red in figure 1 . These figures show also the contours of total density. The subsequent time evolution of these quantities is shown in figure $2(c-j)$. Note that the $y$ position of the $x z$ crosssections (figure $2 b, d, f, h, j$ ) is displaced with time in order to always cut through the mean position of the two vortex centres. At $t=4.4$ (figure $2 c, d$ ), we see that the regions where $0<R i<1 / 4$ and $R i<0$ have widened and intensified but no secondary instability is yet visible. At $t=5$ (figure $2 e, f$ ), Kelvin-Helmholtz rolls are clearly formed in the regions where $0<R i<1 / 4$. These rolls can be seen in both $y z$ and $x z$ cross-sections because they are oblique with respect to the $x$ direction as observed by Deloncle et al. (2008). This creates gravitationally unstable regions as seen by the yellow contours. We stress that the billows in the $x z$ cross-section (figure $2 f$ ) develop in the regions where the condition $R i<1 / 4$ is satisfied because the shear is enhanced and not because the vertical gradient of the total density decreases. In contrast, the overturning of the isopycnals is steeper in the regions where $R i<0$ (red regions, figure $2 f$ ) but no instability is yet apparent there. At $t=6$, the KelvinHelmholtz billows have grown up to a large size (figure $2 g$ ). In the $x z$ cross-section (figure $2 h$ ), there is an abrupt breakdown into small scales leading to large areas of mixed fluid (figure $2 j$ ) as described by Waite \& Smolarkiewicz (2008). However, this breakdown occurs everywhere in the regions where the Richardson number is below $1 / 4$ not only where $R i<0$. In addition, the shear instability develops faster and over a wider region than the gravitational instability. Thus, the breakdown seems mostly due to the Kelvin-Helmholtz instability and not due solely to the gravitational instability as argued by Waite \& Smolarkiewicz (2008). It is likely that there is a coupling between the two instabilities since they develop in neighbouring regions. In particular, the perturbations generated by the shear instability may trigger the gravitational instability. The shear present in the statically unstable regions may also affect the gravitational instability (see Fritts \& Alexander 2003).

Figure 3 shows a similar time series as in figure 2 but for a lower Reynolds number $R e=1000$. For such intermediate values of $R e$, we have observed another scenario 
(a)

z 0.5

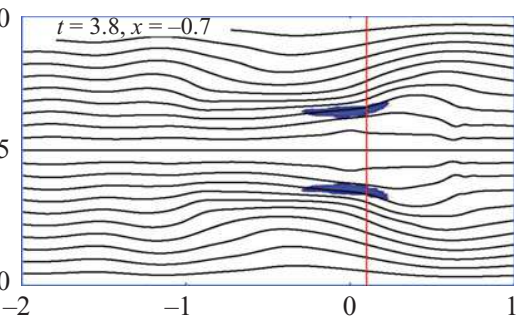

(c)

z 0.5

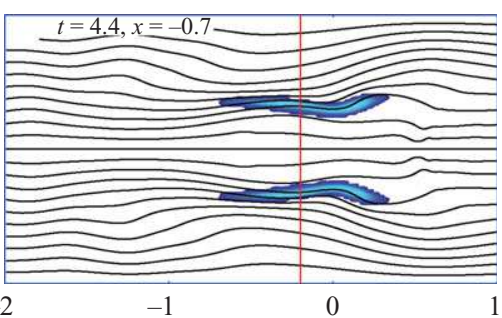

(e)

z 0.5

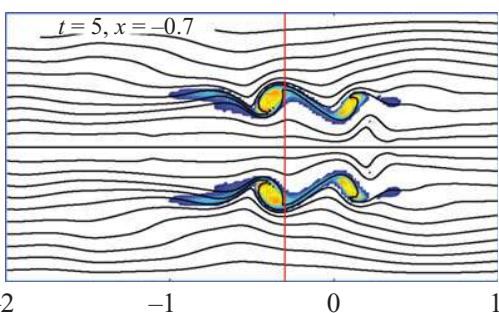

(g)

$z \quad 0.5$

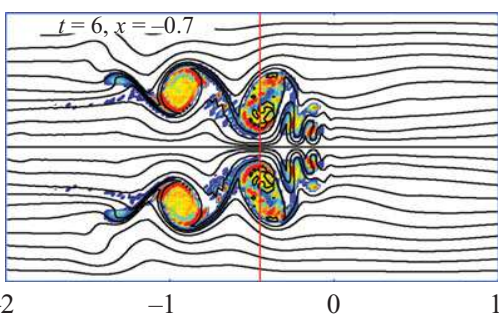

(i)

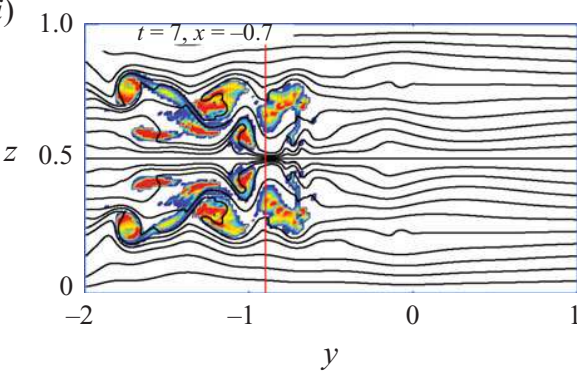

(b)

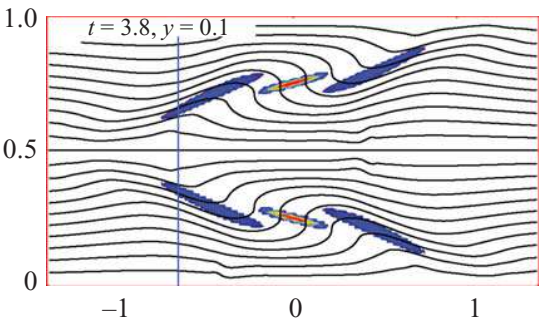

(d)

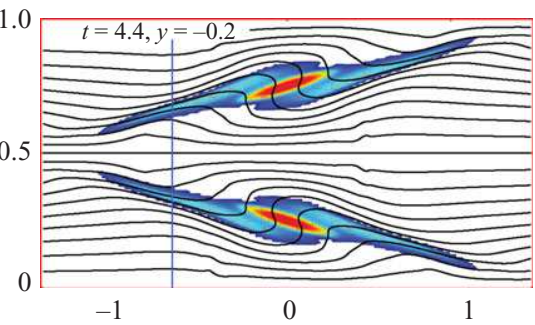

(f)

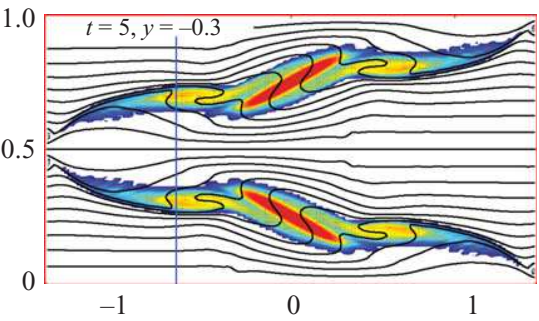

(h)

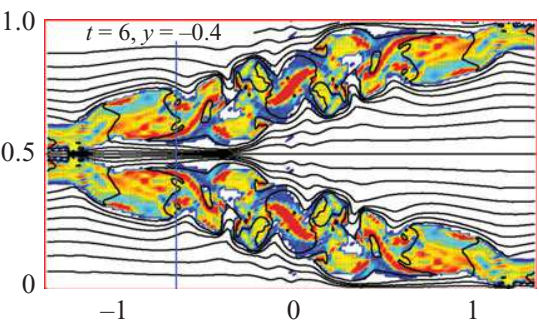

(j)

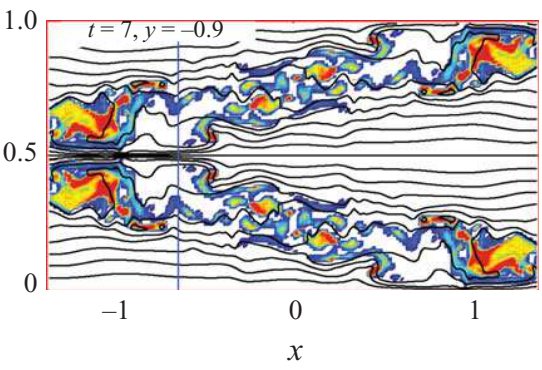

0.25

0.50

FIGURE 2. Time evolution of the total density in the vertical cross-sections $y z(a, c, e, g, i)$ and $x z(b, d, f, h, j)$ indicated in blue and red, respectively, in figure 1 . The colour contours indicate the Richardson number where $R i<1 / 4$. The red vertical lines indicate the location of the $x z$ cross-sections. Conversely, the blue vertical lines indicate the location of the $y z$ cross-sections. The contour interval of total density is equal to $1 / 22$. (Supplementary movie 1, available at journals.cambridge.org/flm.) 

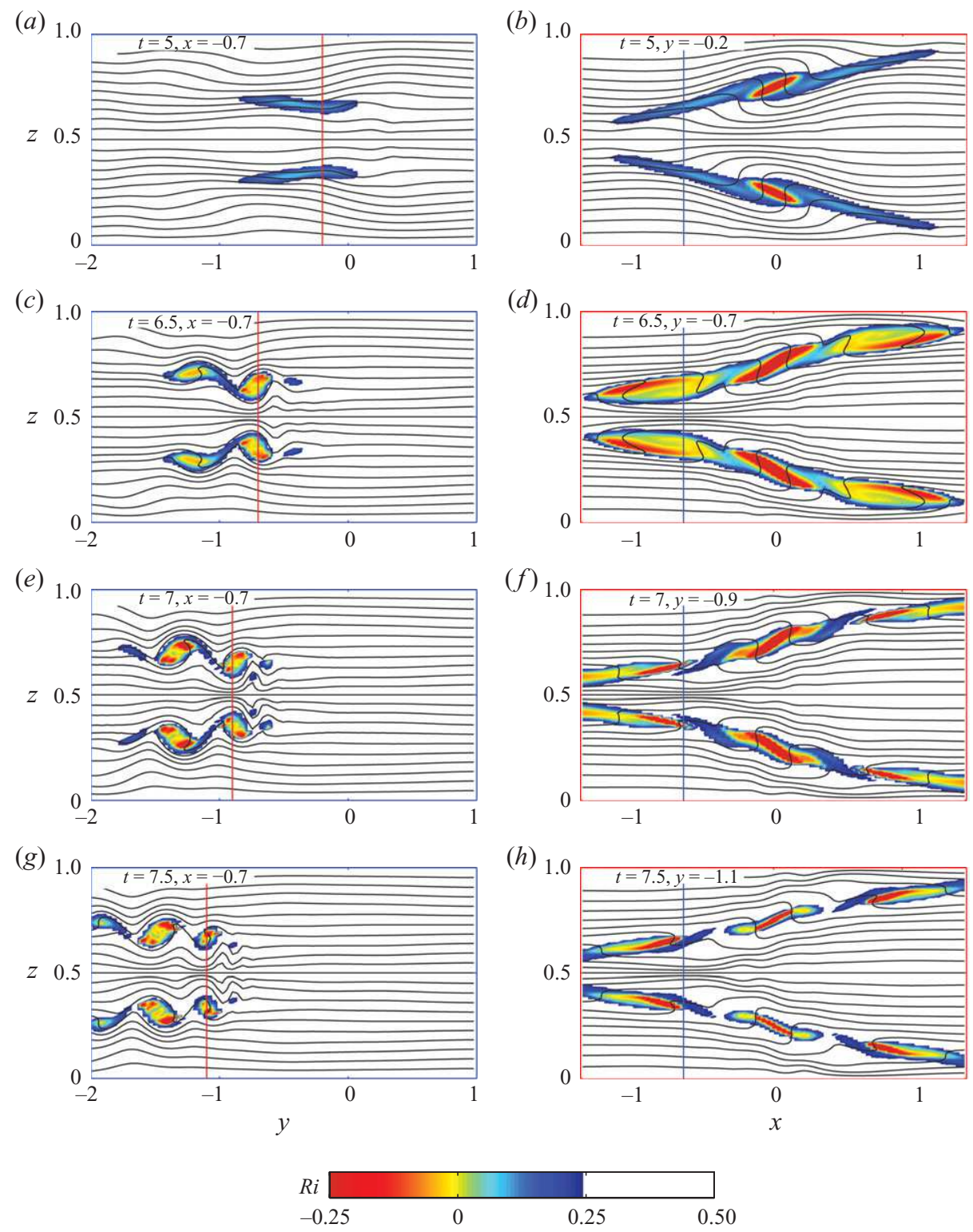

FIGURE 3. Same as in figure 2 but for $R e=1000$. (Supplementary movie 2, available at journals.cambridge.org/flm.)

where only the shear instability operates. Indeed, figure $3(a, b, c, d)$ is very similar to the corresponding figure $2(a, b, e, f)$ except that the Kelvin-Helmholtz billows are less intense. A rolling-up of the isopycnals is also visible in the $x z$ cross-section (figure $3 d$ ) but this occurs in the regions unstable to the shear instability (blue regions at $t=5$, figure $3 b$ ). In contrast, the size of the gravitationally unstable regions (red) near $x=0$ decreases with time after $t=6.5$ (figure $3 d, f, h$ ) without any development of the gravitational instability. Note that the other red regions which appear at $t=6.5$ in figure $3(d, f, h)$ are due to the overturnings created by the Kelvin-Helmholtz billows. 


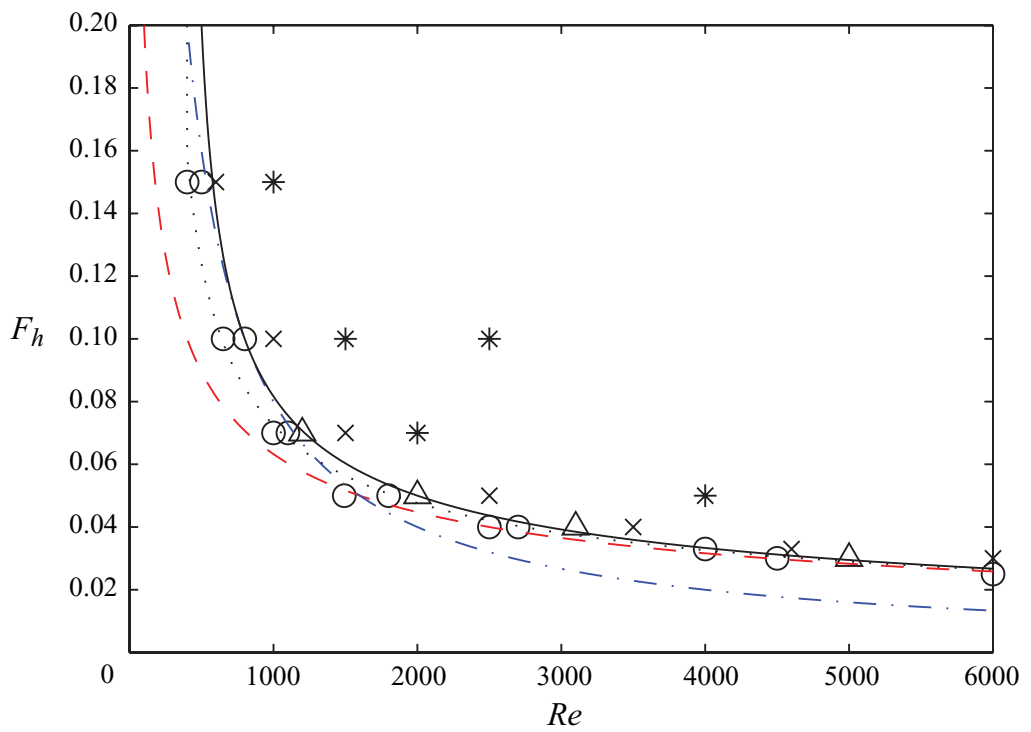

FiguRE 4. (Colour online available at journals.cambridge.org/flm) Summary of all the runs in the parameter space $\left[R e, F_{h}\right]$. The asterisks indicate runs in which both Kelvin-Helmholtz and gravitational instabilities are observed. The crosses correspond to runs where Kelvin-Helmholtz rolls are observed but no gravitational instability is observed. Triangles are intermediate cases for which the shear instability creates undulations but roll-up does not fully develop. The circles denote the runs where no secondary instability is observed. The dashed dotted and dashed lines correspond, respectively, to the criterion $R e F_{h}=80$ and $R e F_{h}{ }^{2}=\mathscr{R}_{c}$, with $\mathscr{R}_{c}=4$. The solid and dotted lines correspond to the criterion $\left(R e-R e_{0}\right) F_{h}{ }^{2}=\mathscr{R}_{c}$, with, respectively, $R e_{0}=400$ and $R e_{0}=240$.

The gravitational instability does not develop probably because its growth rate is not sufficiently high compared to the characteristic time scale of the zigzag instability or compared to the diffusive time scale.

When the Reynolds number is just below the threshold for the onset of secondary instabilities, the minimum Richardson number still drops below $1 / 4$ for a short time period but the shear instability does not appear. It is likely that the growth rate of the shear instability is then too small for the instability to develop over this time period.

\section{Flow regimes in the parameter space $\left[R e, F_{h}\right]$}

Figure 4 summarizes all the different runs in the parameter space $\left[R e, F_{h}\right]$. As already mentioned, only the range $F_{h}<0.2$ has been explored since the zigzag instability is most unstable only in this range in the case of the Lamb-Chaplygin vortex pair (Billant \& Chomaz 2000b). The different symbols distinguish the simulations with gravitational and shear instabilities $(*)$, from those with only the shear instability $(\times)$ and those with no secondary instability $(\circ)$. The triangle symbols $(\triangle)$ indicate an intermediate case for which undulations of the isopycnals are observed but KelvinHelmholtz rolls do not really develop.

The dashed line shows the threshold $R e F_{h}{ }^{2}=\mathscr{R}_{c}$ derived by Deloncle et al. (2008) for the onset of the shear instability. Note that the critical value $\mathscr{R}_{c}$ depends on the properties of the vortex pair and has been empirically found to be $\mathscr{R}_{c} \simeq 4$ in the present case. The alternative threshold $R e F_{h}=80$ proposed by Waite \& Smolarkiewicz 
(a)

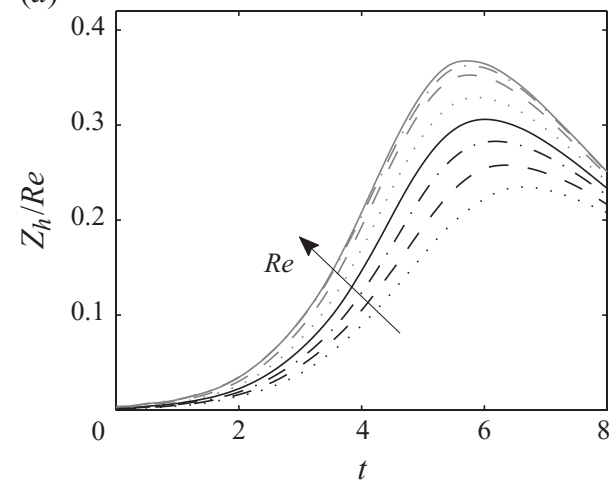

(b)

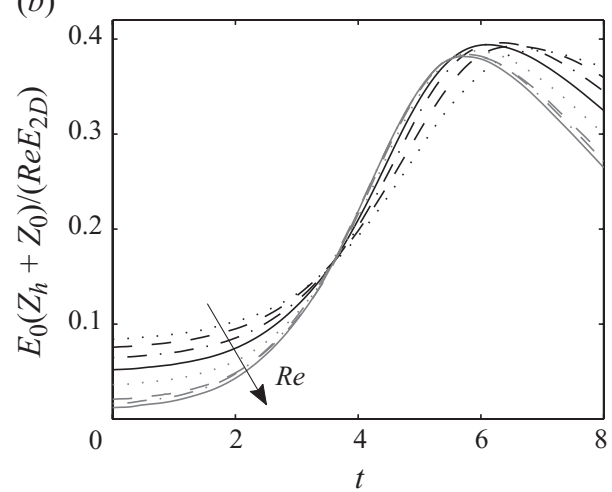

FIGURE 5. Time evolution of $(a)$ the horizontal enstrophy $Z_{h}$ rescaled by $R e$ and of $(b)$ the quantity $\left(Z_{h}(t)+Z_{0}\right) / R e E_{0} / E_{2 \mathrm{D}}(t)$ for different Froude and Reynolds numbers: $(R e=600$, $F_{h}=0.14$, black dotted line $),\left(R e=670, F_{h}=0.12\right.$, black dashed line $),\left(R e=790, F_{h}=0.10\right.$, black dashed dotted line), $\left(R e=1000, F_{h}=0.08\right.$, black solid line $),\left(R e=1480, F_{h}=0.06\right.$, grey dotted line), $\left(R e=2800, F_{h}=0.04\right.$, grey dashed line $),\left(R e=4000, F_{h}=0.03\right.$, grey dashed dotted line) and $\left(R e=6000, F_{h}=0.025\right.$, grey solid line $)$.

(2008) is indicated by the dashed dotted line. We can see that the threshold proposed by Deloncle et al. (2008) works well for large Reynolds number $R e \gtrsim 3000$ but not below. Alternatively, the threshold proposed by Waite \& Smolarkiewicz (2008) is in good agreement for $R e \lesssim 1000$ but departs from the observations for larger $R e$. The solid line shows the threshold $\left(R e-R e_{0}\right) F_{h}{ }^{2}=\mathscr{R}_{c}$, with $R e_{0}=400$, which works remarkably well for any Reynolds number. In particular, it is as good as the threshold $R e F_{h}=80$ for $R e \lesssim 1000$, even if it has not the same functional dependence with $F_{h}$.

This universal threshold can be justified by refining the analysis of Deloncle et al. (2008). These authors observed that the zigzag instability causes an exponential growth of the total horizontal enstrophy per unit of vertical wavelength: $Z_{h}=\left(1 / \mathscr{L}_{z}\right) \int_{\mathscr{r}} \mathrm{d} \mathscr{V}\left|\omega_{h}\right|^{2} / 2$, where $\omega_{h}$ is the horizontal vorticity. This growth saturates only when $Z_{h}$ is of the order of the Reynolds number, i.e. $Z_{h \max } \propto R e$. Since the flow is strongly stratified, the vertical velocity is small and vertical gradients are large so that the horizontal enstrophy $Z_{h}$ is a direct measure of the mean vertical shear of the horizontal velocity, i.e. $Z_{h} \simeq\left(1 / \mathscr{L}_{z}\right) \int_{\mathscr{r}} \mathrm{d} \mathscr{V}\left|\partial \boldsymbol{u}_{h} / \partial z\right|^{2} / 2$. Thus, by assuming that the maximum local shear is proportional to the mean shear, Deloncle et al. (2008) obtained the following estimate for the minimum Richardson number: $R i_{\min } \simeq 1 /\left[F_{h}^{2} \max \left(\left|\partial \boldsymbol{u}_{h} / \partial z\right|^{2}\right)\right] \propto 1 /\left[F_{h}^{2} R e\right]$. Hence, the condition for the shear instability, i.e. $R i<1 / 4$, is equivalent to $R e F_{h}^{2}>\mathscr{R}_{c}$.

The scaling law $Z_{\text {hmax }} \propto R e$ is at the heart of this reasoning. In order to check its validity, we have plotted in figure $5(a)$ the horizontal enstrophy $Z_{h}$ scaled by the Reynolds number for several simulations covering a wide range of parameters: $0.025<F_{h}<0.16$ and $600<R e<6000$. Note that these runs are all below the threshold for the onset of secondary instabilities in order that the development of small scales, which tends to be less affected by the stratification and thus more isotropic, does not introduce any bias on the maximum of the horizontal enstrophy. We see in figure $5(a)$ that all the curves of $Z_{h} / R e$ collapse remarkably well when the Reynolds number is large: $R e \gtrsim 2800$. However, when the Reynolds numbers is 
moderate: $R e \lesssim 1500$, there is a significant decrease of $Z_{h \max } / R e$ when $R e$ decreases and the collapse deteriorates.

In order to understand this discrepancy, it is interesting to consider the equation for the total energy per unit of vertical length in non-dimensional form following Deloncle et al. (2008):

$$
\frac{\mathrm{d} E}{\mathrm{~d} t}=-2 \frac{Z}{R e}-\frac{1}{R e S c} \frac{\int_{\mathscr{V}} \mathrm{d} \mathscr{V}\left|\nabla \rho^{\prime}\right|^{2}}{F_{h}^{2} \mathscr{L}_{z}},
$$

where $Z$ is the total enstrophy per unit of vertical wavelength. The potential energy dissipation (last term in the right-hand side) remains small in all cases (less than $10 \%$ of the total dissipation) so that it can be neglected as a first approximation. The viscous effects (first term in the right-hand side) shown in equation (4.1) will be able to dissipate the energy, i.e. to saturate the development of the zigzag instability, when the total enstrophy is of the order of the Reynolds number: $Z_{\text {max }}=Z_{\text {hmax }}+Z_{v \max }=C R e$, where $C$ is a constant of order unity. Since the vertical enstrophy $Z_{v}$ is observed to decrease from its initial value (which is of order unity by virtue of the nondimensionalization), this implies $Z_{h \max } \sim C R e$ when $R e \gg 1$ (Deloncle et al. 2008). However, for moderate Reynolds number, the accuracy of this approximation is no longer ensured since $Z_{h \max }$ is no longer very large compared to $Z_{\text {vmax }}$. A better estimate is therefore $Z_{h \max } \sim C R e-Z_{0}$, where the vertical enstrophy has been assumed to be approximately constant: $Z_{v \max } \sim Z_{0}$.

In addition, one has to remark that (4.1) is non-dimensionalized based on the initial velocity $U$ and radius $R$ of the dipole. Thus, the relation $Z_{\max } / R e=C$ implicitly assumes that the energy of the dipole $E\left(t_{\max }\right)$ at the time $t_{\max }$ of the enstrophy peak, is approximately the same as at $t=0: E\left(t_{\max }\right) \sim E_{0}$. Again, this assumption is valid for large Reynolds number but is more questionable at moderate Reynolds number. The energy of the dipole indeed significantly decays owing to two-dimensional viscous diffusion before the zigzag instability has reached a finite amplitude. This effect can be taken into account by considering the energy $E_{2 \mathrm{D}}\left(t_{\max }\right)$ that the dipole would have at $t_{\max }$ if the dynamics were purely two-dimensional. This energy can be estimated by $E_{2 \mathrm{D}}(t) \simeq E_{0}-2\left(Z_{0} / R e\right) t$ since the enstrophy $Z_{0}$ remains almost constant for $2 \mathrm{D}$ flows.

Altogether, we see that the relation $Z_{h \max }=C R e$ for $R e \gg 1$ should become for moderate Reynolds number

$$
Z_{\text {hmax }}+Z_{0}=C \operatorname{Re} E_{2 \mathrm{D}}\left(t_{\max }\right) / E_{0}
$$

where the right-hand side has been normalized by $E_{0}$ in order to be consistent with the relation for $R e \gg 1$. In order to test the validity of this scaling law, we show in figure $5(b)$ the quantity

$$
\frac{Z_{h}(t)+Z_{0}}{R e} \frac{E_{0}}{E_{0}-2\left(Z_{0} / \operatorname{Re}\right) t}
$$

for the same set of Froude and Reynolds numbers as in figure 5(a). We see that the maxima of each curve are now almost equal confirming the above reasoning.

Using the relation (4.2) and the assumption $\max \left(\left|\partial \boldsymbol{u}_{h} / \partial z\right|^{2}\right) \propto Z_{h \text { max }}$, it is now a simple matter to deduce that the minimum Richardson number scales like $R i_{\text {min }}=1 /\left[F_{h}^{2} \max \left(\left|\partial \boldsymbol{u}_{h} / \partial z\right|^{2}\right)\right] \propto 1 /\left[F_{h}^{2}\left(R e-R e_{0}\right)\right]$ where $R e_{0}=2 Z_{0} t_{\text {max }} / E_{0}+Z_{0} / C$ is approximately a constant since $t_{\max }$ is almost independent of $R e$. For the LambChaplygin dipole (2.2) in the computational box, we have $Z_{0} \simeq 46.1$ and $E_{0} \simeq 5.5$. Using these values and the empirical values of $C \simeq 0.38$ and $t_{\max } \simeq 7$ obtained from 
figure 5(b), we obtain $R e_{0}^{\prime} \simeq 240$. In figure 4, we see that the criterion $\left(R e-R e_{0}^{\prime}\right) F_{h}^{2}=\mathscr{R}_{c}$ (dotted line) is in better agreement with the data for low $R e$ than the threshold $R e F_{h}^{2}=\mathscr{R}_{c}$. However, an even better agreement can be obtained by using the empirical value $R e_{0}=400$ shown by the black line in figure 4 .

In summary, the constant $R e_{0}$ takes into account two features which were neglected in the analysis of Deloncle et al. (2008): the viscous dissipation of the base flow and the fact that the vertical enstrophy is actually not so small compared to the maximum horizontal enstrophy when the Reynolds number is moderate.

\section{Conclusion}

We have performed direct numerical simulations of the nonlinear development of the zigzag instability of a Lamb-Chaplygin dipole for a large range of Reynolds numbers $R e$ and Froude numbers $F_{h}$ in order to understand the discrepancies between the previous studies of Deloncle et al. (2008) and Waite \& Smolarkiewicz (2008).

Secondary instabilities produce a transition to small-scales when $\left(R e-R e_{0}\right) F_{h}{ }^{2} \geqslant$ $\mathscr{R}_{c}$, where $R e_{0} \simeq 400$ and $\mathscr{R}_{c} \simeq 4$. This threshold, which has been obtained by refining the analysis of Deloncle et al. (2008), is valid for any Reynolds number. It agrees with the threshold derived by Deloncle et al. (2008) for large Reynolds number and with the one of Waite \& Smolarkiewicz (2008) for moderate Reynolds number. Just above the threshold, a shear instability develops in the most bent region of the vortex cores. Further above the threshold, there are simultaneously two types of secondary instabilities: the shear instability mentioned above and a gravitational instability which develops in the middle between the two vortices. However, the shear instability develops faster and seems to play a dominant role in the transition to small scales in all the simulations that have been performed. Our study therefore clarifies and reconciles the different results reported by Deloncle et al. (2008) and Waite \& Smolarkiewicz (2008). Some further simulations have been carried out in the case of two counter-rotating Lamb-Oseen vortices like in Deloncle et al. (2008). They agree qualitatively with the present conclusions and are therefore not shown.

The transition to small scales has important implications for our understanding of stratified turbulence. As pointed out by Deloncle et al. (2008), the shear instability generates small billows whose size $L_{K H}$ scales like the buoyancy length scale $L_{b}=U / N$. There is therefore a direct transfer from the large scales to a small horizontal scale $L_{K H}$ instead of a local cascade. Deloncle et al. (2008) associated $L_{K H}$ to the Ozmidov length scale $l_{o}=\sqrt{\varepsilon / N^{3}}$, where $\varepsilon$ is the mean kinetic dissipation rate per unit mass, because the corresponding Froude number is unity if the typical velocity of the billows is assumed to be $U$, i.e. the same as the velocity of the large-scale vortices. However, it would be more appropriate to call this length scale the buoyancy length scale. As shown recently by Waite (2011), the Ozmidov and buoyancy length scales are indeed distinct physical scales even if they both correspond to a Froude number of order unity. In future investigations it would be interesting to study also the scale selected by the gravitational instability and its implications for stratified turbulence.

We would like to thank A. Deloncle for his invaluable help with the numerical code, D. Guy for technical assistance and the referees for their helpful comments and suggestions. This work is supported by IDRIS (CNRS) for computational facilities under project No. 61722.

Supplementary movies are available at journals.cambridge.org/flm. 


\section{REFERENCES}

Billant, P. 2010 Zigzag instability of vortex pairs in stratified and rotating fluids. Part 1. General stability equations. J. Fluid Mech. 660, 354-395.

Billant, P. \& Chomaz, J.-M. 2000a Experimental evidence for a new instability of a vertical columnar vortex pair in a strongly stratified fluid. J. Fluid Mech. 418, 167-188.

Billant, P. \& Chomaz, J.-M. $2000 b$ Three-dimensional stability of a vertical columnar vortex pair in a stratified fluid. J. Fluid Mech. 419, 65-91.

Billant, P., Deloncle, A., Chomaz, J.-M. \& Otheguy, P. 2010 Zigzag instability of vortex pairs in stratified and rotating fluids. Part 2. Analytical and numerical analyses. J. Fluid Mech. 660, 396-429.

Deloncle, A., Billant, P. \& Chomaz, J.-M. 2008 Nonlinear evolution of the zigzag instability in stratified fluids: a shortcut on the route to dissipation. J. Fluid Mech. 599, 229-238.

Deloncle, A., Billant, P. \& Chomaz, J.-M. 2011 Three-dimensional stability of vortex arrays in a stratified and rotating fluid. J. Fluid Mech. 678, 482-510.

FritTs, D. C. \& AleXander, M. J. 2003 Gravity wave dynamics and effects in the middle atmosphere. Rev. Geophys. 41 (1), 1003.

Howard, L. N. 1961 Note on a paper of John W. Miles. J. Fluid Mech. 10, 509-512.

Lelong, M. P. \& Dunkerton, T. J. 1998 Inertia-gravity wave breaking in three dimensions. Part II: Convectively unstable waves. J. Atmos. Sci. 55 (15), 2489-2501.

MiLEs, J. W. 1961 On the stability of heterogeneous shear flows. J. Fluid Mech. 10, 496-508.

Otheguy, P., Billant, P. \& Chomaz, J.-M. 2007 Theoretical analysis of the zigzag instability of a vertical co-rotating vortex pair in a strongly stratified fluid. J. Fluid Mech. 584, 103-123.

Otheguy, P., Сhомaz, J.-M. \& Billant, P. 2006 Elliptic and zigzag instabilities on co-rotating vertical vortices in a stratified fluid. J. Fluid Mech. 553, 253-272.

Riley, J. J. \& Lelong, M.-P. 2000 Fluid motions in the presence of strong stable stratification. Annu. Rev. Fluid Mech. 32, 613-657.

Waite, M. L. 2011 Stratified turbulence at the buoyancy scale. Phys. Fluids (In press).

Waite, M. L. \& SMolarkiewicz, P. K. 2008 Instability and breakdown of a vertical vortex pair in a strongly stratified fluid. J. Fluid Mech. 606, 239-273. 